\title{
Results of posterior decompression with non-instrumented lumbar fusion surgery in cases of degenerative lumbar canal stenosis presenting with neurogenic claudication in a tertiary care hospital
}

\author{
Maruti Bhujangrao Lingayat, Ansari Muqtadeer Abdul Aziz, Gaurav Balasaheb Mate*, \\ Sourabh Sahebrao Dhamale
}

Department of Orthopaedics, Government Medical College and Hospital, Aurangabad, Maharashtra, India

Received: 26 July 2021

Accepted: 04 August 2021

\section{*Correspondence:}

Dr. Gaurav Balasaheb Mate,

E-mail: gaurav.b.mate@gmail.com

Copyright: ( ) the author(s), publisher and licensee Medip Academy. This is an open-access article distributed under the terms of the Creative Commons Attribution Non-Commercial License, which permits unrestricted non-commercial use, distribution, and reproduction in any medium, provided the original work is properly cited.

\begin{abstract}
Background: Degenerative lumbar canal stenosis remains an important public health problem in today's date. With the overall average age of the world's population rising steadily it is important to have an optimal treatment plan affordable to the masses. Non-instrumented fusion after decompression remains an important treatment option which is affordable to the masses and effectively treats the instability occurring due to degeneration process. This study aims to understand the results of such treatment in a tertiary care center catering to the masses.

Methods: The study was conducted in 34 patients with diagnosed degenerative lumbar canal stenosis with neurogenic claudication who underwent decompressive laminectomy with a posterolateral strut graft posterolateral fusion from July 2018 to August 2020. Each patient was followed up for 12 months.

Results: In the present study, a total of 34 patients with degenerative lumbar canal stenosis with neurogenic claudication were included. There were 18 male and 16 female patients. The pre-operative Swiss spinal stenosis Score was 61-80 $(52.9 \%)$ and these scores improved to a majority of patients in the category of 21-40 (82.4\%) at 1 year postoperatively. The average VAS score was $4.7 \pm 1.8$ preoperatively while the average post op VAS score was $0.8 \pm 0.77$.

Conclusions: Non-instrumented fusion of the vertebrae with decompression has significantly improved results at 1 year follow up postoperatively and it is an excellent easy and cost-effective technique if used in a properly selected patient. Further studies are required to assess its long-term results.
\end{abstract}

Keywords: Degenerative lumbar canal stenosis, Posterior decompression, Non-instrumented fusion

\section{INTRODUCTION}

During the last four to five decades extensive research has focused attention on the syndrome of lumbar canal stenosis that is now on the rise. ${ }^{1}$ Lumbar canal stenosis is a medical condition in which the spinal canal becomes narrow (stenosis occurs) thereby compressing the nerves at the level of the lumbar vertebrae. ${ }^{2}$ Various causes lead to this severely debilitating condition of which most common is spinal degeneration occurring with aging besides others. ${ }^{3,4}$

Lumbar canal stenosis is most common in the elderly age group, beginning at around 50 years and rapidly rising above 65 years of age. Up to $80 \%$ of people above 70 years of age have lumbar canal stenosis. ${ }^{5}$ It is important to treat this condition accurately and in such a way that patient gets relief with treatment modality that will suit him/her. Many treatment options are available today for the condition. ${ }^{6}$

Treatment options are decided based on radiographic findings, clinical features and the choice and fitness of the patient for surgery. However, with such a plethora of options available for treating the patient one is often confused as to which is right for a particular patient. Owing to these factors it is important to define adequate factors as baseline for a particular modality and also to decide which 
treatment plan will be better than the other in a particular patient. $^{7}$

The topic of this study is the surgical modality of noninstrumented fusion post decompression. It offers the patient the advantages of affordability and avoiding outside implants in the body along with stabilizing the spine. With the advent of instrumented techniques noninstrumented techniques have been falling behind and their importance is being ignored. In a tertiary care hospital catering to the masses, resources are limited and many a times implants are limited in availability. The main aim of this study therefore is to focus on this treatment modality as an important option to the patients suffering from lumbar canal stenosis.

\section{METHODS}

This study was conducted after getting ethical clearance from July 2018 to August 2020 and all the patients who fulfilled the inclusion criteria were included in the study. All the patients were followed up for up to 12 months postoperatively. The diagnosis of degenerative lumbar canal stenosis was made based on clinical symptoms, physical signs, laboratory findings, and radiological evidence.

Patients were assessed clinically; a thorough history and complete physical examination was done. The subjective symptoms and objective signs were recorded in a predetermined format. This was then followed by preoperative routine investigations as well as MRI lumbar spine to confirm the diagnosis (Figure 1).

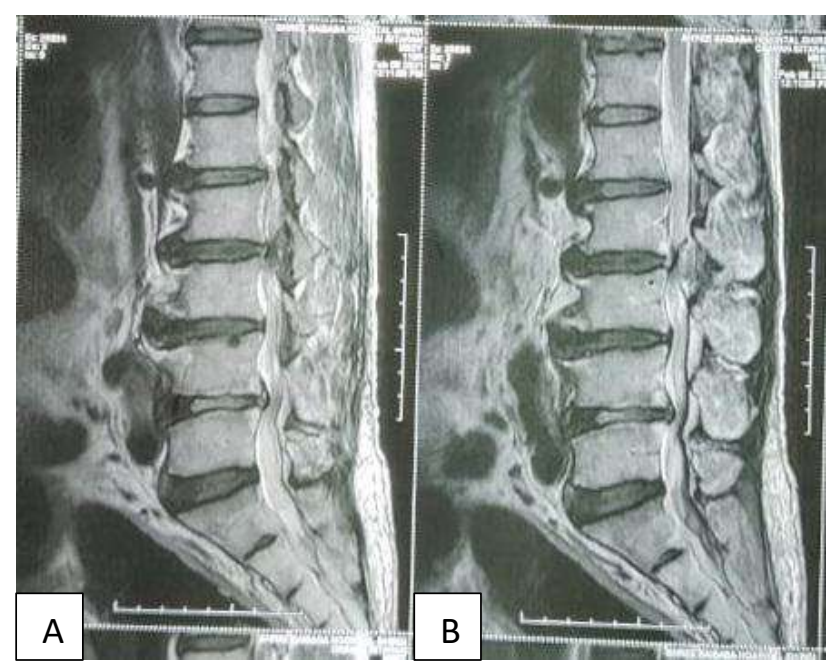

Figure 1 (A and B): MRI of canal stenosis at L2-L3 and L4-L5 level.

General work-up of the patient was done (Complete blood count, liver function test, kidney function test, blood sugar level, prothrombin time, arterial Doppler of the affected limb, serology testing, 2-D echocardiography) along with any specific investigations if advised by physician and anesthetist.
Pre-op assessment using Swiss spinal stenosis scale and the visual analogue scale (VAS) was done. ${ }^{8-10}$ Scales used for lumbar canal stenosis are subjective and depend a lot on patient's physical and mental condition.

\section{Sample size}

Sample size included in the study was 34 patients.

\section{Study design}

The study design was prospective interventional study.

\section{Study period}

The study conducted from July 2018 to August 2020, for duration of 2 years and 2 months.

The Swiss spinal stenosis scale is a disease-specific selfreport outcome instrument commonly used in trials to measure treatment outcomes in patients with lumbar spinal stenosis (LSS). It consists of 3 sections in total. The first 2 sections are used in the preoperative evaluation and in total consist of 12 questions. The last section is used to measure post-op outcome. The Swiss spinal stenosis questionnaire consists of 3 sections:

- Symptom severity scale (questions I-VII) [further subdivided into pain domain (questions I-IV) and a neuro-ischemic domain (questions V-VII)]: Possible range of the score is 1 to 5 .

- Physical function scale (questions VIII-XII): Possible range of scores is 1-4.

- Patient's satisfaction with treatment scale (questions XIII-XVIII): range of score is 1-4.

All patients underwent adequate laminectomy with clearing of the lateral recess (foraminotomy) and then a non-instrumented posterolateral fusion with a strut graft from iliac crest. Findings were noted in detail.

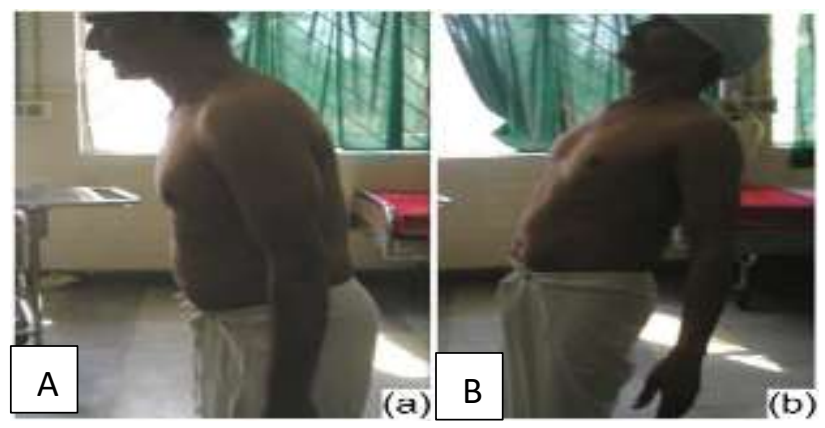

Figure 2: (A) Pre-operative patient needed to flex his spine to relieve symptoms. (B) Patient able to walk comfortably with spine extended.

Patients were discharged at around 4 days after surgery on average. Regular follow up was done at 3 weeks, 3 months, 6 months and one year. Patients were assessed for presence 
of back pain and neurological deficit at each visit and the postoperative results were recorded in the respective scales at 1-year post-op (Figure 2).

\section{Operative procedure}

All patients were operated under general anesthesia. The patient was positioned prone with padding done over the chest and hips with hips placed in extension and knees flexed to decrease nerve tension. Intravenous antibiotic (injection linezolid 600 milligrams intravenously) was administered 30 minutes prior to incision. Shaving of skin hair over surgical site was done 10 minutes before surgery and Foley's catheterization was done. Painting of surgical site was done with betadine scrub $(7.5 \%)$ followed by draping of surgical site. The involved intervertebral disc was visualized with fluoroscopy and marked. A standard dorsal midline incision was taken over the marked disc extending 5 centimeters proximally and distally. Dissection carried down in the midline through the skin, subcutaneous tissue and fascia to the tip of spinous process, the muscles were detached subperiosteally in a caudal to cephalad direction to expose the posterior elements. Spinous processes at the affected level were removed and laminectomy was done. Foraminotomy was performed and the nerve root was decompressed. Bone graft obtained from the iliac crest was placed in between the transverse processes on either side. An uncharged drain was kept and the wound was closed in layers covered by adequate dressing.

\section{Post-operative care}

Post-operative X-rays were taken. Patient was kept in intensive care unit (ICU) after surgery for one day for observation of vital parameters. Foley's catheter was removed after 12 hours. Drain was removed after 12-24 h. Intravenous antibiotics were given for 3 days followed by 5 days of oral antibiotics. Patients were advised to wear a brace till bony fusion was evident on $\mathrm{x}$-rays.

\section{RESULTS}

In this study 34 patients with degenerative lumbar canal stenosis with neurogenic claudication between the age group of 50-70 years were operated with decompression laminectomy and non-instrumented posterolateral fusion from July 2018 to August 2020.

In this study, out of 34 patients maximum (12) patients were from the age group of 66-70 years while there were only 6 patients from the age group of 50-55 years. The youngest patient was of 50 years whereas oldest was of 70 years. The mean age was 61 years (Figure 3 ).

Out of 34 patients in the study, 47 percent (16) patients were female while 53 percent (18) were male. The male to female ratio was 1.13:1 (Figure 4).
Majority of these patients under study had undergone some form of conservative treatment in the past and were considered for operative when their symptoms did not resolve. $55.9 \%$ patients had symptoms between 6 to 10 months, $35.3 \%$ patients had symptoms between 11 to 15 months while $8.8 \%$ patients had symptoms more than 16 months (Figure 5).

In this study the canal stenosis was most common at L4L5 level with up to 50 percent patients having the same. Second most common level was L3-L4 while the least common level was L1-L2 (Figure 6).

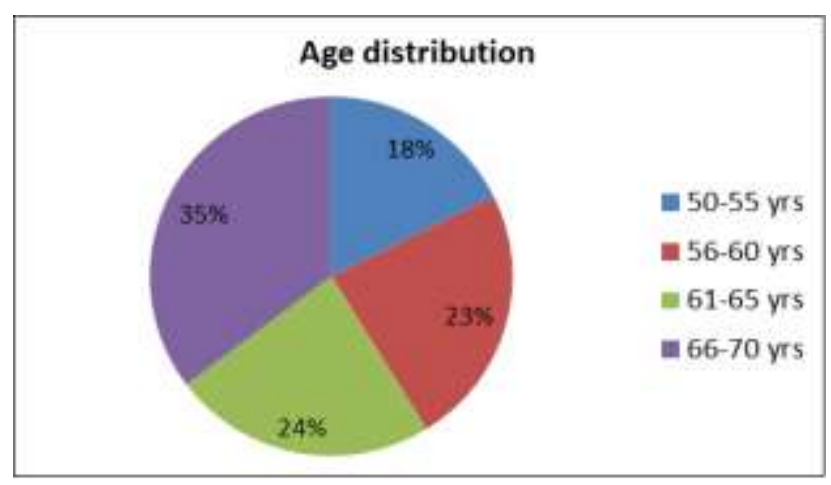

Figure 3: Age distribution of patients in the study.

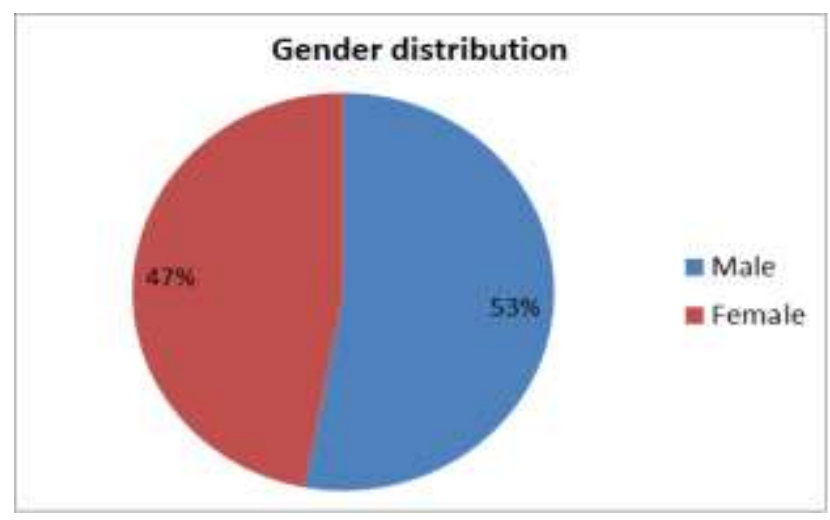

Figure 4: Gender distribution of patients in the study.

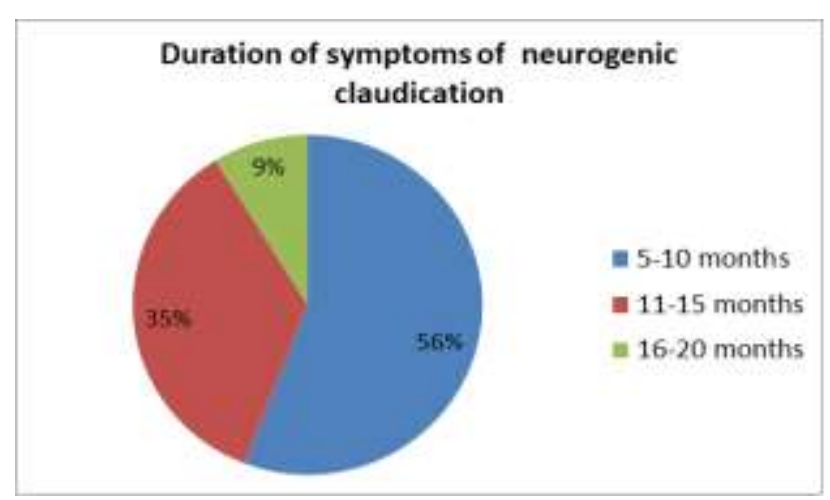

Figure 5: Duration of symptoms of neurogenic claudication of patients in the study. 


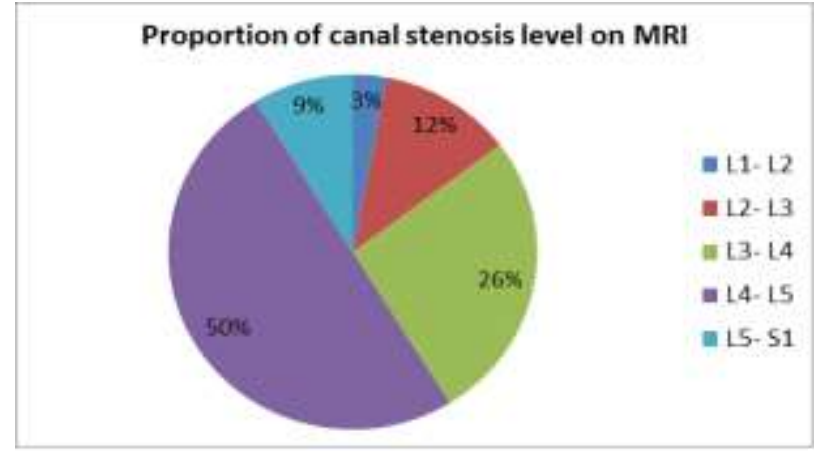

Figure 6: Distribution of level of stenosis in the study.

Out of the 34 patients, 7 had a preop VAS score of 1-3, 22 had a VAS score of 4-6 and 5 patients had a VAS score of
7-10. The average preop VAS score was 4.7 with a standard deviation of 1.8. At 1 year follow-up 15 had a postop VAS score of 0 (i.e., pain free) and 19 patients had a VAS score of 1-3. The average postop VAS score was 0.8 with a standard deviation of 0.77 . The average preop VAS score was 4.7 while the average post op VAS score was 0.8 (Table 1$)$.

The pre-op and post-op Swiss spinal stenosis severity questionnaire results were compared in the test and the results obtained were as given below. Majority of the patients were having a score of 61-80 (52.9\%) and after surgery these scores improved to a majority of patients coming in the category of $21-40(82.4 \%)$ at 1 year postoperatively. In SSS score the lesser percentage obtained, the better is the patient's result (Table 2).

Table 1: The VAS scores grouped for an easy understanding.

\begin{tabular}{|lllll|}
\hline VAS score & Pre-operative & Percentage $\mathbf{( \% )}$ & $\begin{array}{l}\text { Post-operative } \\
\text { No. of cases }\end{array}$ & Percentage (\%) \\
\hline $\mathbf{0}$ & No. of cases & 0 & 15 & 44.1 \\
\hline $\mathbf{1 - 3}$ & 0 & 20.6 & 19 & 55.9 \\
\hline $\mathbf{4 - 6}$ & 7 & 64.7 & 0 & 0 \\
\hline $\mathbf{7 - 1 0}$ & 22 & 14.7 & 0 & 0 \\
\hline Total & 5 & 100 & 34 & 100 \\
\hline
\end{tabular}

Table 2: Swiss spinal stenosis scale analysis.

\begin{tabular}{|lllll|}
\hline $\begin{array}{l}\text { Swiss spinal stenosis } \\
\text { score (lesser the } \\
\text { better) }\end{array}$ & Pre-operative & Post-operative & Percentage (\%) \\
\hline $\mathbf{0 - 2 0}$ & 0 & 0 & No. of cases & 0 \\
\hline $\mathbf{2 1 - 4 0}$ & 2 & 5.9 & 0 & 82.4 \\
\hline $\mathbf{4 1 - 6 0}$ & 13 & 38.2 & 6 & 17.6 \\
\hline $\mathbf{6 1 - 8 0}$ & 18 & 52.9 & 0 & 0 \\
\hline $\mathbf{8 1 - 1 0 0}$ & 1 & 2.9 & 0 & 0 \\
\hline Total & 34 & 100 & 34 & 100 \\
\hline
\end{tabular}

\section{DISCUSSION}

Laminectomy is a commonly done procedure for lumbar canal stenosis with neural claudication. The presence of neurogenic claudication denotes that the disease has progressed to such an extent that it is necessary to operate it rather than to try for conservative line of management. Various conservative practices have been applied to patients of lumbar canal stenosis but when claudication sets in, it is necessary for an aggressive intervention to be done. Laminectomy effectively decompresses the compressed structures but leads to instability of the vertebral column due to the gap created in bony continuum. Non-instrumented posterolateral fusion was derived as an effective counter to this instability. An iliac crest graft is biological and there is no fear of increased risk of infection as compared to instrumented fusion. It also negates the cost associated with the use of pedicle screws and rods/other forms of instrumented fixation. There is also no need for second surgery for removal of implants as in the instrumented fixation procedures. The present study analyzed the results of this surgical technique on the basis of functional and clinical outcomes of the patients. The Swiss spinal stenosis scale is an important parameter for understanding the effectiveness. The advantage of the Swiss spinal stenosis scale is that its wide parameters of evaluation give a better understanding as compared to other indices.

In the present study, the age group of study was 50 to 70 years with the mean age being 61 years. Maximum patients in the study $(35.3 \%)$ were in the age group of $66-70$ years. The Framingham study found that the prevalence increases with age. In patients $<40$ years, the prevalence of relative and absolute LSS was $20.0 \%$ and $4.0 \%$, respectively; in those 60-69 years the prevalence was $47.2 \%$ and $19.4 \%$, respectively. ${ }^{11}$

Prasad et al in their study there were a total of $22(45.83 \%)$ male and $26(54.16 \%)$ female patients (total patients being 
48). ${ }^{12}$ In the present study there were $18(53 \%)$ male and $16(47 \%)$ female patients with the total patients being 34 . There was slightly more incidence of male patients in our study. This could be attributed to regional variation.

In the present study the most commonly affected level was L4-5 (50\%) followed by L3-4 level (26.5\%). This is similar to the results obtained by Prasad et al of the L4-5 level $(47 \%)$ followed in incidence by the L3-4 $(25.77 \%)$ and the L5-S1 $(23.71 \%)$ level. ${ }^{12}$ Lumbar canal stenosis occurs most commonly at the L4-5 level. These levels seem to be the most frequently affected by mechanical stress.

In the present study $19(55.9 \%)$ patients had symptoms less than 10 months, $12(35.3 \%)$ patients had symptoms between 11 to 15 months while $3(8.8 \%)$ patients had symptoms more than 16 months. This is comparable to the results of analysis of the SPORT trial by Radcliffe et al (2012) where they found that 227 (35.9\%) patients had duration more than 12 months and $405(64.1 \%)$ patients had symptoms less than 12 months. ${ }^{13}$ A prolonged duration of symptoms was associated with poorer outcomes post operatively.

In the present study, the average preop VAS score was $4.7 \pm 1.8$ while the average post op VAS score was $0.8 \pm 0.77$. Prasad et al had an average pre-operative VAS score (back pain) of $7.96 \pm 0.619$ and post-operative VAS score (back pain) of $3.81 \pm 0.0762 .{ }^{12}$ In both studies there was a significant relief obtained from pain relative to the preoperative baseline.

The Swiss spinal Stenosis scores were also significantly improved postoperatively with average pre-operative being $64 \pm 12.42$ and postoperatively $33.3 \pm 7.9$.

\section{CONCLUSION}

Although our study has certain limitations due to various factors it can be concluded that non instrumented fusion has indeed promising results at 1 year postoperatively and it is economical, has low requirement of a second surgery and earlier return to work with quick recovery. The outcomes are better when patient is operated as early as possible if conservative management has failed and neurogenic claudication sets in. Proper patient selection is very important. Non-instrumented fusion with laminectomy done in properly selected patients after thorough evaluation achieves rewarding and gratifying results. The Swiss spinal Severity score is an excellent scale to assess the patients of spinal stenosis. Its advantage is that it measures in 3 criteria with one of them being the evaluation specifically pertaining to post op patient satisfaction. Such an evaluation is lacking in many other scales for spinal stenosis such as the Oswestry disability index or the Fukushima LSS scale. Significant improvement was obtained in the SSS scores postoperatively. There was a significant improvement in VAS of the patients after operation. The VAS is perhaps the best universal score which can be used due to its ease of access and simplicity. All in all, non-instrumented fusion of the vertebrae with decompression has significantly improved results at 1 year follow up postoperatively and it is an excellent easy and costeffective technique if used in a properly selected patient. Further studies are required to assess its long-term results.

\section{ACKNOWLEDGEMENTS}

The authors are grateful to all the patients, colleagues and hospital staff without whom this study would not have been possible.

\section{Funding: No funding sources}

Conflict of interest: None declared

Ethical approval: The study was approved by the institutional ethics committee

\section{REFERENCES}

1. Wu A, March L, Zheng X, Huang J, Wang X, Zhao J, Blyth FM, Smith E, Buchbinder R, Hoy D. Global low back pain prevalence and years lived with disability from 1990 to 2017: estimates from the Global Burden of Disease Study 2017. Ann Transl Med. 2020;8(6):299.

2. Munakomi S, Foris LA, Varacallo M. Spinal Stenosis And Neurogenic Claudication. [Updated 2020 Jul 4]. In: StatPearls. Treasure Island (FL): StatPearls Publishing; 2020.

3. Billy GG, Lemieux SK, Chow MX. Changes in Lumbar Disk Morphology Associated With Prolonged Sitting Assessed by Magnetic Resonance Imaging. PM\&R, 2014;6:790-5.

4. Ishimoto Y, Cooper C, Ntani G. Factory and construction work is associated with an increased risk of severe lumbar spinal stenosis on MRI: A case control analysis within the wakayama spine study. Am J Ind Med. 2019;62:430-8.

5. Wu AM, Zou F, Cao Y, Xia DD, He W, Zhu B, et al. Lumbar spinal stenosis: an update on the epidemiology, diagnosis and treatment. AME Med J. 2017;2:63.

6. Jackson RP, McManus AC, Moore J. Lumbar spinal stenosis: treatment options for an aging population. Mo Med. 2012;109(6):466-9.

7. Garfin SR, Fischgrund JS, Eismont FJ, Bono CM, Bell GR. Rothman-Simeone and Herkowitzes The Spine. Edn- Seventh, Elsevier, China, 2018, 10381057.

8. Abou-Al-Shaar H, Adogwa O, Mehta AI. Lumbar Spinal Stenosis: Objective Measurement Scales and Ambulatory Status. Asian Spine J. 2018;12(4):76574.

9. Pratt RK, Fairbank JC, Virr A. The reliability of the Shuttle Walking Test, the Swiss Spinal Stenosis Questionnaire, the Oxford Spinal Stenosis Score, and the Oswestry Disability Index in the assessment of 
patients with lumbar spinal stenosis. Spine (Phila Pa 1976). 2002;27(1):84-91.

10. Comer CM, Conaghan, PG, FRACP, FRCP*, $†$; Tennant, Alan PhD. Internal Construct Validity of the Swiss Spinal Stenosis Questionnaire, Spine. 2011;36(23):1969-76.

11. Kalichman L, Cole R, Kim DH, Li L, Suri P, Guermazi A, Hunter DJ. Spinal stenosis prevalence and association with symptoms: the Framingham Study. Spine J. 2009;9(7):545-50.

12. Prasad BC, Ramesh Chandra VV, Devi BV, Chivukula SS, Pundarikakshaiah K. Clinical, radiological, and functional evaluation of surgical treatment in degenerative lumbar canal stenosis. Neurol India. 2016;64(4):677-83.

13. Radcliff K, Hilibrand A, Lurie JD, Tosteson TD, Delasotta L, Rihn J, Zhao W, Vaccaro A, Albert TJ,
Weinstein JN. The impact of epidural steroid injections on the outcomes of patients treated for lumbar disc herniation: a subgroup analysis of the SPORT trial. J Bone Joint Surg Am. 2012;94(15):1353-8.

Cite this article as: Lingayat MB, Aziz AMA, Mate GB, Dhamale SS. Results of posterior decompression with non-instrumented lumbar fusion surgery in cases of degenerative lumbar canal stenosis presenting with neurogenic claudication in a tertiary care hospital. Int J Res Orthop 2021;7:926-31. 